\title{
DOIS MODELOS MATEMÁTICOS CAPAZES DE SOLUCIONAR UM PROBLEMA PERIÓDICO DE MANUTENÇÃO ATRAVÉS DE ROTEAMENTO EM ARCOS
}

\author{
Guilherme Vinicyus Batista \\ Universidade Federal do Paraná \\ guivbatista89@gmail.com \\ Cassius Tadeu Scarpin \\ Universidade Federal do Paraná \\ cassiusts@gmail.com
}

\begin{abstract}
Resumo
Problemas envolvendo manutenção, em grande parte das vezes, exigem intervenções periódicas programadas segundo uma frequência. No caso de rodovias e ferrovias há uma espécie de monitoramento e inspeção durante determinados intervalos de tempo com o objetivo de garantir o fluxo nas vias. O objetivo desse artigo é apresentar e comparar um resultado de dois modelos matemáticos para o Problema de Roteamento em Arcos Capacitado e Periódico (PCARP). Essa classe de problemas têm ganhado mais atenção na última década, nela precisa-se determinar uma rota para um ou múltiplos veículos respeitando suas capacidades, considerando um horizonte discreto de tempo de forma que as demandas de cada arco sejam atendidas. Os resultados encontrados são satisfatórios ao aplicados nesse contexto de manutenção.
\end{abstract}

Palavras-Chaves: Manutenção; Modelos Matemáticos; PCARP; Periódico; Roteamento em Arcos.

\begin{abstract}
Problems involving maintenance, in most cases, require scheduled periodic interventions according to a frequency. In the case of roads and railways, there is a kind of monitoring and inspection during certain time intervals in order to ensure the flow on roads. The aim of this paper is to present and compare a result of two mathematical models for the Periodic Capacitated Arc Routing Problem (PCARP). This class of problems has gained more attention in the last decade, where it must be determined a route to one or multiple vehicles respecting their capacities, considering a discrete time horizon so that the demands of each arc are attended. The results are satisfactory when applied in this context maintenance.

Keywords: $\quad$ Maintenance; Mathematical Models; PCARP; Periodic; Arc Routing;
\end{abstract}




\section{INTRODUÇÃO}

Muitos problemas logísticos, como localização de facilidades, gerenciamento de armazéns, entre outros têm sido resolvidos por pesquisadores. Os quais têm tomado uma sequência de resolução envolvendo decisões estratégicas, táticas e operacionais [7]. Outro exemplo que vêm sendo solucionado da mesma forma são os problemas de roteamento de veículos que podem ser divididos em duas classes: roteamento em nós e roteamento em arcos.

O roteamento em nós consiste em encontrar uma ou mais rotas onde um veículo visita alguns ou todos os nós de um grafo e o roteamento em arcos tem por objetivo determinar uma rota que passa por todos ou alguns arcos ou arestas de um grafo [13]. Diferentemente dos problemas de roteamento em nós, que são os problemas de roteamento mais estudados, a literatura do problema de roteamento em arcos é mais pobre e desorganizada, fato relatado em Ghiani et al. [5] que trata do problema de gerenciamento de lixo, uma das principais aplicações do problema de roteamento em arcos.

Esses problemas se distinguem basicamente na modelagem em virtude da natureza dos serviços, passagens em arcos ou nós. Segundo Assad e Golden [1] o roteamento em arcos ocorre quando existe uma alta densidade de pontos em uma linha, sendo convertido de um problema de roteamento em nós para arcos, ou simplesmente quando o interesse é atravessar arcos.

Entre alguns fatores listados na literatura que ampliam a diversidade dos problemas de roteamento encontra-se: número de domicílios, tempo para servir um determinado nó ou arco, tamanho da frota, natureza da demanda, localização da demanda, restrições na capacidade dos veículos, natureza do grafo, entre outros [9].

Dependendo da forma como o problema é tratado pode ocorrer uma série de complicações para sua resolução. O objetivo desse artigo é apresentar e comparar dois modelos matemáticos capazes de resolver problemas inseridos no contexto de manutenção preditiva de ferrovias ou rodovias, onde a cada intervalo de tempo algumas vias necessitam ser inspecionadas a fim de evitar possíveis acidentes e garantir o fluxo contínuo de veículos.

Esse tipo de problema é classificado como Problema de Roteamento em Arcos Capacitado e Periódico (PCARP), na próxima seção é apresentada um breve levantamento bibliográfico e a definição desse problema. Um problema é sugerido e contextualizado na seção 3 afim de exemplificar uma situação de PCARP, o mesmo será resolvido por dois modelos matemáticos propostos na seção 4, porém como os modelos atendem objetivos diferentes uma adaptação feita para solucionar problema é explicada na mesma seção. Em seguida está listado o resultado na seção 5 e finalmente as conclusões em 6.

\section{O PROBLEMA DE ROTEAMENTO EM ARCOS CAPACITADO E PERIÓDICO}

Os Problemas de Roteamento em Arcos, do inglês Arc Routing Problems (ARP), têm por objetivo determinar o menor custo para atravessar um conjunto de arcos de um grafo, com ou sem restrições. Essa classe de problemas pode ser aplicada numa série de contextos práticos como coleta de lixo, entrega de cartas, remoção de neve, inspeção em linhas de energia elétrica, inspeção, monitoramento e manutenção de ferrovias e rodovias.

Ao contextualizar os ARP obtêm-se algumas variações, uma delas é o Roteamento em Arcos Capacitado e Periódico, Periodic Capacitated Arc Routing Problem (PCARP), o mesmo trata de veículos com capacidade limitada que devem, ao decorrer de horizonte de tempo bem definido, fazer uma rota atendendo a demanda de cada arco. Demanda essa que é geralmente estabelecida em forma de frequência.

Corberán e Prins [4], trazem em seu trabalho um apanhado dos resultados 
encontrados na literatura, durante a década que precede sua publicação, sobre os Problemas de Roteamento em Arcos que tiveram origem no Problema das Pontes de Königsberg e que tentam encontrar uma ou mais rotas que cubram todas (ou parcialmente) as ligações (arcos ou arestas) de um grafo, satisfazendo algumas restrições e com o menor custo possível. Os problemas tem apresentado variações que o tornam cada vez mais complexos de serem resolvidos.

Quando se trata de ARP um dos principais problemas encontrados na literatura é o Capacitated Arc Routing Problem (CARP), Problema de Roteamento em Arcos Capacitado. Ele é caracterizado por ter uma demanda não negativa associada a cada arco do grafo. Dado que cada veículo de uma frota tem uma determinada capacidade, e esses veículos devem atravessar os arcos coletando ou entregando as determinadas demandas sem excede-la, problema este proposto por Golden e Wong em 1981 [6].

O CARP é amplamente utilizado para resolver o problema da coleta de lixo urbano como uma decisão de nível operacional, tomada diariamente. Porém em alguns problemas é necessário tomar uma decisão de nível tático, onde envolva um horizonte de tempo maior com múltiplos períodos, sujeito a restrições que envolvem determinadas frequências, partindo dessas características foi proposto o PCARP por Lacomme, Prins e Ramdane-Chérif [10] que apresentam este problema, suas variantes e um Algoritmo Genético para resolvê-lo.

De maneira geral, o PCARP é uma extensão natural do CARP onde o problema é resolvido para múltiplos períodos ao invés do problema ser resolvido para apenas um dia [13]. Resolver o PCARP implica em determinar simultaneamente as decisões em nível tático e operacional durante um horizonte de tempo. Infelizmente, em muitas aplicações desse problema, existem algumas complicações, como por exemplo: as demandas podem ser flutuantes de acordo com os dias ou espaçadas em restrições entre dias de serviço [11].

Lacomme, Prins e Ramdane-Chérif [11] propõe uma definição básica para o PCARP, bem como alguns algoritmos para sua resolução. Dado um grafo e um discreto horizonte de tempo $H$ com $n p$ períodos, cada arco tem uma frequência $f(a)$ que pode ser de no máximo uma vez por dia, restrição essa que ainda pode ser expressa com base no intervalo entre serviços no mesmo arco. O mesmo autor ainda afirma: "o PCARP implica na determinação simultânea das decisões no nível tático e operacional em todo horizonte de tempo” (página 539).

Em muitas aplicações o PCARP tem particularidades que o tornam mais complexo, como demandas flutuantes ao longo do tempo ou ainda restrições de intervalos entre dias de serviço (periodicidades ou frequências). Baseado nisso o problema foi classificado em duas classes [11] como ilustra a Figura 1.

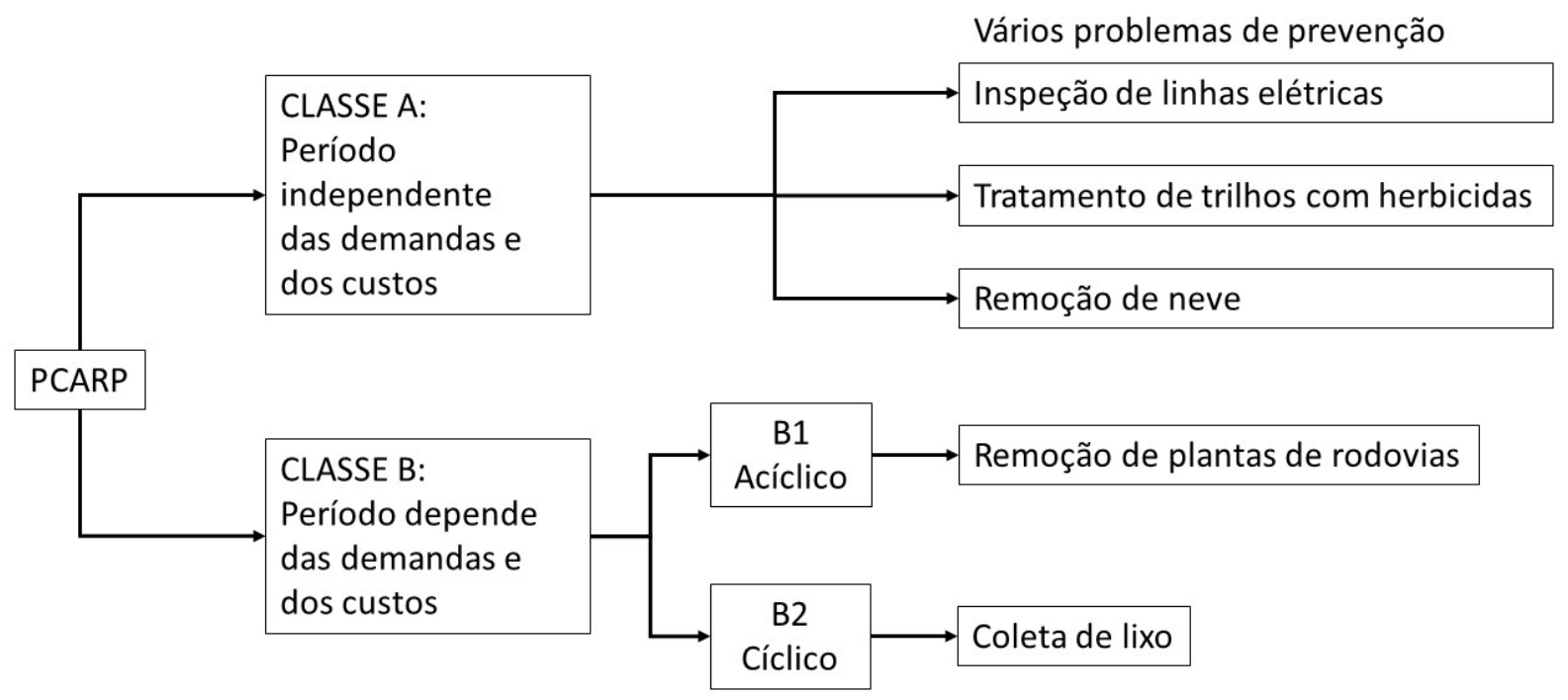


Figura 1: Uma simples classificação do PCARP. Fonte: Lacomme et al. [11]

A CLASSE A representa o conjunto dos problemas em que a demanda não resulta de acumulação diária de algum fator, por exemplo, muitos serviços de manutenção ou ainda de prevenção, inspeção e monitoramento, como o serviço de pulverização de herbicidas em trilhos de trem onde o tempo gasto por metro e a demanda ao longo do tempo é fixa. Já a CLASSE B envolve problemas como a coleta de lixo, onde a demanda aumenta conforme vão se passando os dias. Além disso, a CLASSE B pode ser subdivida em problemas acíclicos (subclasse B1) ou cíclicos (subclasse B2). Em B1, o horizonte de tempo é bem definido e a demanda é flutuante ao longo do tempo, como no caso da remoção de plantas em rodovias, assumindo que no inverno as plantas não crescem de forma que a demanda é nula. Em B2 o intervalo de tempo se repete continuamente, por exemplo, a coleta de lixo se repete de semana em semana [11].

Chu, Labadi, \& Prins [3] foram os primeiros a propor uma modelagem matemática para o PCARP onde também foi proposto lower bounds prelimares e uma metaheurística chamada Scatter Search. Eles conseguiram resultados ótimos em softwares comerciais apenas para instâncias muito pequenas com 5 períodos e 10 arcos, por exemplo, fato já esperado devido à natureza do problema - NP-Hard. Em [2] os mesmos autores propuseram três heurísticas capazes de resolver o problema. Kansou e Yassine [8] combinaram um algoritmo da colônia de formigas com uma heurística de inserção para resolver o PCARP conseguindo resultados robustos e com uma performance rápida.

\section{PROPOSTA DE PROBLEMA}

Os serviços de manutenção são de extrema importância para empresas. A manutenção é um investimento que proporciona uma redução não somente nos custos de reparo de equipamentos como no custo de paradas do processo [12]. Marcorin e Lima [12] definem manutenção preventiva como um conjunto de ações que visam prevenir a quebra e está associada à intervenções periódicas geralmente programadas segundo uma frequência. A manutenção preventiva acarreta muitas vezes em intervenções desnecessárias e troca de peças, enquanto as preditivas caracterizam-se por uma manutenção de acompanhamento, medição e análise de variáveis que possam prognosticar uma eventual falha exigindo uma mão-de-obra mais qualificada e alguns aparelhos ou instrumentos de medição.

Um exemplo de problema que pode ser resolvido como PCARP, mais especificamente da CLASSE A, é o problema da Figura 2, nela está representada uma malha ferroviária. Nessa malha ferroviária deve ser feita uma manutenção preditiva, realizada por um veículo qualificado para o serviço, capaz de fazer as medições necessárias. Alguns trechos devem ser visitados uma vez a cada 16 dias enquanto outros uma vez a cada 24 dias, afim de serem inspecionados evitando possíveis acidentes. 


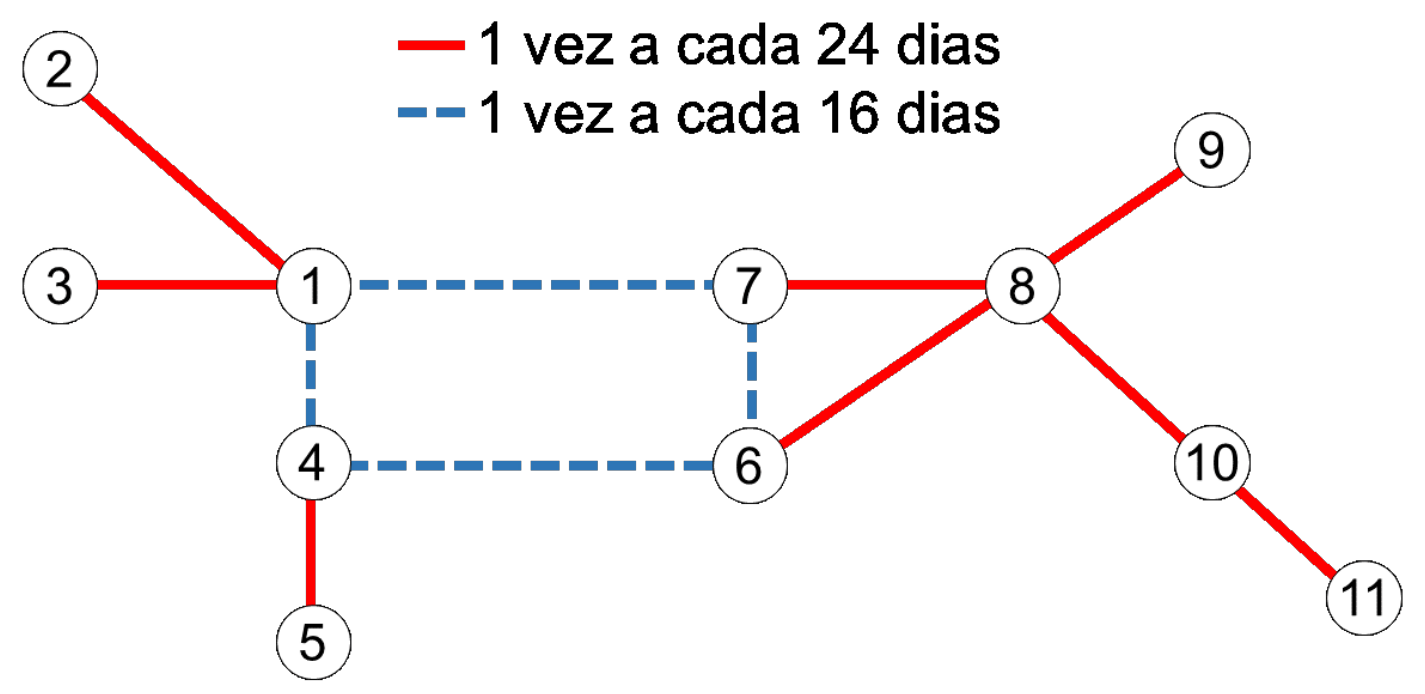

Figura 2 - Representação de um problema de inspeção em uma malha ferroviária

A distância entre um ponto à outro ponto ligado por um mesmo arco na Figura 2 é medida em tempo e é equivalente a 1 dia de serviço ou travessia. Por exemplo, demora-se um dia para sair do ponto 1 e deslocar-se até o ponto 7, trabalhando ou apenas atravessando. Algumas das característica desse problema proposto é o fato de que o deslocamento dos veículos, em geral, é lento e ao final de um dia não é necessário voltar a um ponto específico (também conhecido como depósito).

O horizonte de tempo é definido baseado no arco de maior periodicidade. Entende-se por periodicidade o intervalo de tempo em que um arco deve ser atendido ao menos uma vez. No caso do exemplo da Figura 2 temos uma horizonte de tempo de 24 dias. Ou seja, dentro do período de 24 dias os arcos tem que ter sua demanda atendida. Isso implica que os arcos com periodicidade de 16 dias devem ter duas passagens durante esses 24 dias, de forma que o intervalo entre uma passagem e outra seja de no máximo 15 dias, ou ainda, que em 16 dias o trecho seja percorrido ao menos uma vez.

Como é um problema cíclico, ao final dos 24 dias o carro deve estar no mesmo ponto onde iniciou seu trajeto no dia 1, o que é equivale a dizer que o que deve ocorrer no dia 25 é exatamente o que ocorre no primeiro dia. Arcos com menor periodicidade devem levar em conta a sequência dos dias após o final dos primeiros 24 dias. Por exemplo, se o carro passou no arco 3-7 durante os dias 3 e 9 ele teria atendido a demanda dos primeiros 24 dias, porém como o dia 3 seria equivalente ao dia 27 , após fechado o primeiro ciclo ele não atenderia ao próximo.

\section{MODELOS MATEMÁTICOS}

Monroy, Amaya e Langevin, (2013) [13] modela matematicamente um problema de monitoramento da malha rodoviária, que é realizado periodicamente, e propõe uma heurística para sua resolução. As ruas ou rodovias são divididas em classes de acordo com a necessidade de vigilância e a hierarquia das vias, essas classes servem como base para determinar as necessidades (número de passagens) de cada arco ao longo do horizonte de tempo. O objetivo é designar um conjunto de rotas, uma para cada carro em cada dia, satisfazendo as frequências de cada classe de vias em cada sub período de tempo sem exceder a capacidade do veículo.

O modelo proposto por Monroy, Amaya and Langevin é feita para grafos direcionados onde tem-se um depósito localizado no ponto 1 , as notações que utiliza para formular seu problema são as seguintes:

$\Omega_{c}$ : conjunto de arcos da classe $\boldsymbol{c}$ 
$\boldsymbol{T}$ : conjunto dos conjuntos $\Omega_{c}$

$\boldsymbol{e}_{\boldsymbol{c}}$ : conjunto dos subperíodos (sub horizontes) associados a arcos da classe $\boldsymbol{c}$

$\boldsymbol{O}(\boldsymbol{n})$ : conjunto dos arcos deixando o nó $\boldsymbol{n} \in \boldsymbol{N}$

$\boldsymbol{I}(\boldsymbol{n})$ : conjunto dos arcos entrando no nó $\boldsymbol{n} \in \boldsymbol{N}$

$\boldsymbol{S}$ : subconjunto de arcos

$\boldsymbol{N}(\boldsymbol{S})$ : conjunto dos nós incidentes nos arcos de $\boldsymbol{S}$

$\boldsymbol{V}$ : conjunto de rotas

$\boldsymbol{w}_{\boldsymbol{j}}$ : conjunto de rotas correspondentes ao subperíodo $\boldsymbol{j}$

$\boldsymbol{f}_{\boldsymbol{j}}$ : número de serviços requeridos no subperíodo $\boldsymbol{j}$

$\boldsymbol{p}_{\boldsymbol{a}}$ : peso do arco $\boldsymbol{\alpha}$

Em suas instâncias, utiliza um horizonte de tempo de 14 dias e 3 tipos de classes. A classe 1 é formada pelos arcos que tem necessidades de ao menos uma passagem trabalhando nos dias 1 até 5, 6 até 7, 8 até 12 e 13 até 14, a classe 2 tem demandas entre os dias 1 até 7 e 8 até 14, e a classe 3 é formada pelos arcos que necessitam de passagem entre o dia 1 até o dia 14. A Figura 3 a seguir ilustra essa situação:

\begin{tabular}{|c|c|c|c|c|c|}
\hline & $\begin{array}{l}\text { Classe de } \\
\text { arcos }\end{array}$ & $\begin{array}{ll}\text { Segunda } & - \\
\text { Sexta (1) }\end{array}$ & $\begin{array}{l}\text { Sábado - } \\
\text { Domingo (1) }\end{array}$ & $\begin{array}{ll}\text { Segunda } & - \\
\text { Sexta (2) }\end{array}$ & $\begin{array}{l}\text { Sábado - } \\
\text { Domingo (2) }\end{array}$ \\
\hline \multirow{3}{*}{ Subperíodos } & CLASSE 1 & $w_{1}$ & $w_{2}$ & $w_{3}$ & $w_{4}$ \\
\hline & CLASSE 2 & \multicolumn{2}{|c|}{$w_{5}$} & \multicolumn{2}{|c|}{$w_{6}$} \\
\hline & CLASSE 3 & \multicolumn{4}{|c|}{$w_{7}=V$} \\
\hline
\end{tabular}

[13].

Figura 3 - Sub horizontes de tempo associados a tipos de arcos. Fonte: Monroy et al.

Sua formulação consiste em definir k rotas que atendam as demandas, as variáveis de decisão utilizam notação de arcos e são as seguintes:

servido)

$x_{a}^{k}$ assume o valor 1 se o arco $a$ é servido pela rota $k$ ou então 0 caso contrário

$y_{a}^{k}$ contabiliza o número de vezes que o arco $a$ é atravessado pela rota $k$ (sem ser

O problema é modelado da seguinte forma:

$\max z=\sum_{k \in V} \sum_{a \in R} p_{a} x_{a}^{k}$

Sujeito à:

$\sum_{a \in O(n)}\left(x_{a}^{k}+y_{a}^{k}\right)-\sum_{a \in I(n)}\left(x_{a}^{k}+y_{a}^{k}\right)=0 \forall k \in V, n \in N$
$\sum_{a \in R}\left(c_{a} x_{a}^{k}\right)+\sum_{a \in R}\left(t_{a} y_{a}^{k}\right) \leq Q \forall k \in V$
$M \sum_{a \in I(N(S))}\left(x_{a}^{k}+y_{a}^{k}\right) \geq \sum_{a \in O(N(S))} x_{a}^{k}+y_{a}^{k} \forall S \subset A, 1 \notin N(S), k \in V$

$\sum_{k \in w_{j}} x_{a}^{k} \geq f_{j} \forall j \in e_{o}, a \in \Omega_{0}, c=1, \ldots,|T|$

$x_{a}^{k} \in\{0,1\} \forall a \in R, k \in V$

$y_{a}^{k} \in Z^{+} \forall a \in A, k \in V$

Quanto mais importante o arco, mais passagens ele deve receber durante o intervalo de tempo, portanto a função objetivo (1) maximiza o número de serviços levando em conta a classe de cada arco e seu respectivo peso. A conservação do fluxo dos veículos é garantida por (2). Cada rota que será feita por um carro e é delimitada por uma capacidade (3), sendo que a capacidade total é a soma dos custos de atravessar $t_{\alpha}$ e servir o arco $c_{\alpha}$. As restrições de 
conectividade são dadas em (4). A relação entre o número de serviços por cada período e a classe de arcos é dada na equação (5). E as restrições (6) e (7) definem as variáveis.

Um modelo proposto para resolver especificamente o problema sugerido parte de um grafo não direcionado $G=(X, E)$ com $n$ pontos, logo, $X=\left\{x_{1}, x_{2}, \ldots, x_{n}\right\}$, e $m$ arestas com $E=\left\{e_{1}, e_{2}, \ldots, e_{m}\right\}$ que devem ser percorridos por $n k$ carros definidos pelo conjunto $K=\{1,2, \ldots, k\}$. Cada arco $e$ é formado por um par de nós, e é chamado de $x_{i j}=\left(x_{i j}, x_{j}\right)$ ou $[i, j]$ e é associado a um custo $c_{i j}$.

A demanda ou periodicidade de cada arco é expressa na quantidade máxima de períodos em que o arco deve ser atendido ao menos uma vez $M P\left(x_{i j}\right)$. Tem-se ainda o horizonte de tempo $H$, formado por $n p$ períodos, onde cada período é simbolizado pela letra $p$. O modelo é baseado em Programação Linear Binária, logo para a resolução do problema foram criadas três variáveis binárias apresentadas abaixo:

$x_{i j k p}$ assume o valor 1 se o arco o carro $k$ se desloca do ponto $i$ para $i$ no período $p$

$p_{i j p}$ assume valor 1 se o arco $x_{i j}$ não respeita a periodicidade no período $p$

$f_{i k p}$ assume valor 1 se o carro $k$ fica parado no ponto $i$ no dia $p$

As variáveis $p_{i j p}$ e $f_{i k p}$ foram criadas para que o problema tenha uma viabilidade. Cada variável $p_{i j p}$ tem associado a ela um custo $P U_{i j}$, que é uma punição caso a periodicidade de um arco não seja atendida. Isso permite que na modelagem proposta haja um atraso no atendimento de alguns arcos, visto que a quantidade deve ser a menor possível. A variável $f_{i k p}$ permite que o carro folgue em um determinado dia. A função objetivo dada pela equação (8) busca minimizar os custos de deslocamento e evitar que atrasos ocorram, então problema é modelado da seguinte maneira:

$\min Z=\sum_{[i, j] \in E} \sum_{k=1}^{n k} \sum_{p=1}^{n p} c_{i j} * x_{i j k p}+\sum_{[i, j] \in E} \sum_{p=1}^{n p} P U_{i j} * p_{i j p}$

Sujeito à:

$\sum_{[i, j] \in E} x_{i j k p}+f_{j k p}-\sum_{[i, j]]_{E}} x_{j i k, p+1}-f_{j k, p+1}=0 \quad \forall j \in X, k, p$

$\sum_{[i, j] \in E} x_{i j k p}+\sum_{[i, j] \in E} x_{j i k p}+\sum_{i=1}^{n} f_{i k p}=1 \forall p, k$

$\sum_{k=1}^{n k}\left(\begin{array}{c}x_{i j k p}+x_{j i k p}+x_{i j k, p+1}+x_{j i k, p+1}+\ldots \\ +x_{i j k, p+M P\left(x_{i j}\right)-1}+x_{j i k, p+M P\left(x_{i j}\right)-1}\end{array}\right)+p_{i j p} \geq 1 \quad \forall[i, j] \in E, p$

$x_{i j k p}, p_{i j p}, f_{i k p} \in\{0,1\} \quad \forall[i, j] \in E, k, p$

A restrição (9) garante o fluxo diário dos carros permitindo a folga, sendo que, como serão formados ciclos, ao fim do período $n p$ o carro deve retornar para o mesmo local em que estava no dia 1, ou seja, o período $n p+1$ é igual ao dia 1 , e assim por diante. Isso vale para todo o horizonte de tempo e para todas as restrições. A restrição (10) garante que todos os carros terão alguma designação para o dia $p$, além disso, garante implicitamente que a capacidade de cada carro não seja excedida.

Já a restrição (11) é a mais complexa. Ela se refere à periodicidade que deve ser atendida em cada trecho. Na restrição (11) é possível que as variáveis $p_{i j p}$ assumam valor 1 , ou seja, um arco pode ser atrasado em um dia ao custo de ser punido com um custo $P U_{i j}$. Contudo, se a necessidade for de apenas uma passagem durante todo o horizonte de tempo, a restrição pode ser simplificada para apenas um dia $p, p=1$, o que abrange todo o horizonte de tempo e a variável $p_{i j p}$ pode ser descartada, como feito no problema proposto. Finalmente, 
a equação (12) determina que todas as variáveis sejam binárias. O fato de não conter um depósito não obriga o carro partir de nenhum ponto, porém todos serão atravessado se possível e da melhor maneira.

\subsubsection{Adaptação do modelo}

As duas modelagens abordadas atendem diferentes escopos, para efeitos comparativos entre os modelos foi feita uma adaptação ao modelos proposto por Monroy, Amaya, e Langevin [13] onde:

$\square$ Foi considerado um horizonte de tempo de 24 dias que foram subdividos em 2 grupos de dias baseado nos dias de cada periodicidade. Então obteve-se o primeiro grupo envolvendo os dias entre 1 e 16, e o outro grupo com os dias 17 a 24 ;

$\square$ O grafo foi considerado não direcionado;

$\square$ A função objetivo foi definida para minimizar o custo das viagens;

$\square$ Como a modelagem proposta por Monroy et al. necessita de uma depósito, foi escolhido o ponto 1 pois ele está conectado a arcos com maior número de necessidades (1-7, 1-4);

Foram definidas duas classes de arcos. A primeira classe, Classe 1, necessita de 2 passagens no horizonte de tempo em $w_{1}, w_{2}$ que são formados respectivamente pelos dias 1 a 16 e 17 a 24 . A Classe 2 necessita apenas uma passagem no intervalo $w_{3}$ que engloba todo o horizonte de tempo. A Figura 4 abaixo exemplifica as classes:

\begin{tabular}{|l|c|c|}
\cline { 2 - 3 } \multicolumn{1}{c|}{} & Dias 1-16 & Dias 17 - 24 \\
\hline Classe 1 & $w_{1}$ & $w_{2}$ \\
\hline Classe 2 & \multicolumn{2}{c|}{$w_{3}$} \\
\hline
\end{tabular}

Figura 4 - Sub horizontes de tempo associados a tipos de arcos para adaptação

Fez-se necessário a criação de duas rotas, a primeira com uma capacidade de 16 que englobava os 16 primeiros dias, e outra com capacidade de 8 que englobava os dias 17 até 24, que no futuro formarão uma rota única que deve atender de maneira aproximada as periodicidades;

$\square$ As restrições que evitam subciclos foram simplificadas apenas considerando o os subconjuntos de arcos que fizessem com que o depósito sempre fosse incluído nas rotas. No trabalho proposto por Monroy, Amaya, e Langevin foi feita uma simplificação semelhante sendo que se a rota apresentasse subciclos então eram acrescentadas restrições que evitavam as mesmas.

\section{RESULTADO}

Com a adaptação feita, o modelo de Monroy et al. chegou a uma solução exata, a qual apresentou as seguintes rotas para o problema da Figura 2 apresentado na seção II: 1 - 7 6 - 8 - 9 - 8 - 10 - 11 - 10 - 8 - 6 - 4 - 1 - 2 - 1 - 3 - 1 e 1 - 7 - 8 - 7 - 6 - 4 - 5 - 4 - 1, a primeira rota englobando os primeiros 16 dias e a seguinte os próximos 8 dias. De maneira geral, é equivalente dizer que ao longo dos 24 dias a rota obtida foi 1 - 7 - 6 - 8 - 9 - 8 - 10 - 11 - 10 8 - 6 - 4 - 1 - 2 - 1 - 3-1 - 7 - 8 - 7 - 6 - 4 - 5 - 4 - 1 .

Observa-se que a solução obtida é interessante, porém não satisfaria a resolução do problema, pois ao avaliar os arcos que tem necessidade maior que 1 passagem ao longo do horizonte de tempo ocorre uma falha no atendimento, percebe-se que: 
$\square$ O arco 1-7 é atendido nos dias 1 e 17, dentro do limite máximo da periodicidade pois o arco é atravessado exatamente 16 dias depois da primeira passagem. Contando a característica de ser um problema cíclico ele seria atravessado no dia 25 novamente que seria após 8 dias depois da última passagem;

O arco 6-4 é atendido nos dias 11 e 21, e futuramente no 35 também dentro das periodicidades;

$\square$ Orco 1-4 é atendido nos dias 12 e 24, e futuramente no 36 também dentro das periodicidades;

$\square$ O arco 7-6 é atendido nos dias 1 e 20, o que não atenderia a periodicidade pois entre os dias 2 a 19 não é atravessado, teria um novo atendimento no dia 25 porém extrapolou da primeira vez.

De fato, no modelo especifico se não fosse possível atender os arcos dentro da periodicidade ocorreria uma punição no arco 7-6 por atrasos e a solução seria aceita. Porém, o modelo específico apresentou a seguinte a rota ótima para o mesmo problema: 6 - 4 - 1 - 7 - 8 - 10 - 11 - 10 - 8 - 7 - 6 - 4 - 5 - 4 - 1 - 2 - 1 3 - 1 - 7 - 6 - 8 - 9 - 8 - 6 onde nenhum arco seria atrasado. A Tabela 1 a seguir lista as duas soluções com destaque para os arcos críticos que necessitam de mais de uma passagem ao longo do horizonte de tempo.

Tabela 1 - Solução apresentada ao resolver os modelos

\begin{tabular}{|c|c|c|c|c|c|}
\hline \multirow{2}{*}{ Dia } & \multicolumn{2}{|c|}{$\begin{array}{c}\text { Modelo de Monroy et al. } \\
\text { (2013) }\end{array}$} & \multicolumn{2}{c|}{ Modelo Específico } \\
\cline { 2 - 6 } & $\begin{array}{c}\text { Ponto de } \\
\text { partida }\end{array}$ & $\begin{array}{c}\text { Ponto de } \\
\text { chegada }\end{array}$ & Rota & $\begin{array}{c}\text { Ponto de } \\
\text { partida }\end{array}$ & $\begin{array}{c}\text { Ponto de } \\
\text { chegada }\end{array}$ \\
\hline 1 & 1 & 7 & 1 & 6 & 4 \\
\hline 2 & 7 & 6 & 1 & 4 & 1 \\
\hline 3 & 6 & 8 & 1 & 1 & 7 \\
\hline 4 & 8 & 9 & 1 & 7 & 8 \\
\hline 5 & 9 & 8 & 1 & 8 & 10 \\
\hline 6 & 8 & 10 & 1 & 10 & 11 \\
\hline 7 & 10 & 11 & 1 & 11 & 10 \\
\hline 8 & 11 & 10 & 1 & 10 & 8 \\
\hline 9 & 10 & 8 & 1 & 8 & 7 \\
\hline 10 & 8 & 6 & 1 & 7 & 6 \\
\hline 11 & 6 & 4 & 1 & 6 & 4 \\
\hline 12 & 4 & 1 & 1 & 4 & 5 \\
\hline 13 & 1 & 2 & 1 & 5 & 4 \\
\hline 14 & 2 & 1 & 1 & 4 & 1 \\
\hline 15 & 1 & 3 & 1 & 1 & 2 \\
\hline 16 & 3 & 1 & 1 & 2 & 1 \\
\hline 17 & 1 & 7 & 2 & 1 & 3 \\
\hline 18 & 7 & 8 & 2 & 3 & 1 \\
\hline 19 & 8 & 7 & 2 & 1 & 7 \\
\hline 20 & 7 & 6 & 2 & 7 & 6 \\
\hline 21 & 6 & 4 & 2 & 6 & 8 \\
\hline 22 & 4 & 5 & 2 & 8 & 9 \\
\hline 23 & 5 & 4 & 2 & 9 & 8 \\
\hline & & & & & \\
\hline
\end{tabular}




\section{CONCLUSÃO}

O fato do modelo específico não conter nenhuma atraso, mostra que os objetivos foram atingidos de uma forma mais eficaz do que utilizando uma adaptação do modelo proposto em [13], outro fator considerável é que o modelo de Monroy et al. teve uma solução mais rápida computacionalmente para esse experimento. Ao rodar os modelos no software CPLEX 12.4 em um computador Intel Core i7 com 2 Ghz de processamento e 4 Gb de memória RAM e sistema operacional 64 bits, o modelo de Monroy et al. foi resolvido em menos de 1 segundo (importante ressaltar da simplificação feita para evitar subciclos e que nenhum subciclo foi formado na primeira tentativa) enquanto o modelo especifico demora pouco menos de 30 segundos para chegar em uma solução com o valor ótimo, porém, esse valor só é confirmado após 330 segundos. Considerando que é uma solução para um planejamento a longo prazo, esses valores se tornam irrelevantes ao comparar com 24 dias.

Os problemas de roteamento em arcos abrangem um campo de atuação ainda não tão explorado e têm grande aplicabilidade a contextos reais podendo ser adaptados conforme as necessidades. É muito relevante fazer um planejamento de serviços que não se restrinjam a apenas um dia e abranjam um horizonte de tempo mais longo, bons resultados podem ser alcançados e acidentes podem ser prevenidos como no caso apresentado.

\section{REFERÊNCIAS BIBLIOGRÁFICAS}

[1] Assad, A.A. and Golden, B.L. 1995. Arc Routing Methods and Applications. Handbooks in Operations Research and Management Science, volume 8, Network Routing. 375-483.

[2] Chu, F., Labadi, N. and Prins, C. 2005. Heuristics for the periodic capacitated arc routing problem. Journal of Intelligent Manufacturing. 16, 2 (2005), 243-251.

[3] Chu, F., Labadi, N. and Prins, C. 2004. THE PERIODIC CAPACITATED ARC ROUTING PROBLEM LINEAR PROGRAMMING MODEL, METAHEURISTIC AND LOWER BOUNDS. Journal of Systems Science and Systems Engineering. 13, 4 (2004), 423-435.

[4] Corberán, A. and Prins, C. 2010. Recent Results on Arc Routing Problems : An Annotated Bibliography. Networks. (2010), 50-69.

[5] Ghiani, G., Laganà, D., Manni, E., Musmanno, R. and Vigo, D. 2014. Operations research in solid waste management: A survey of strategic and tactical issues. Computers \& Operations Research. 44, (2014), 22-32.

[6] Golden, B.L. and Wong, R.T. 1981. Capacitated Arc Routing Problem. Networks. 11, (1981), 305-315.

[7] Hashemi Doulabi, S.H. and Seifi, A. 2013. Lower and upper bounds for location-arc routing problems with vehicle capacity constraints. European Journal of Operational Research. 224, 1 (Jan. 2013), 189-208. 
[8] Kansou, A. and Yassine, A. 2009. Ant Colony System for the Periodic Capacitated Arc Routing Problem Problem. Proc. 2009 International Network Optimization Conference (2009), 1-7.

[9] Konowalenko, F. 2012. PROBLEMA DO CARTEIRO CHINÊS NÃO-ORIENTADO E MISTO PARA A OTIMIZAÇÃO DE ROTAS NA CIDADE DE IRATI / PR. Universidade Federal do Paraná.

[10] Lacomme, P., Prins, C. and Ramdane-Chérif, W. 2002. Evolutionary Algorithms for Multiperiod Arc Routing Problems. 9th Int. Conf. on Information Processing and Management of Uncertainty in Knowledge-Based systems (Annecy, France, 2002), 1-8.

[11] Lacomme, P., Prins, C. and Ramdane-Chérif, W. 2005. Evolutionary algorithms for periodic arc routing problems. European Journal of Operational Research. 165, (2005), 535-553.

[12] Marcorin, W.R. and Lima, C.R.L. 2003. Análise dos Custos de Manutenção e de Nãomanutenção de Equipamentos Produtivos. Revista de Ciência \& Tecnologia. 11, 22 (2003), 35-42.

[13] Monroy, I.M., Amaya, C.A. and Langevin, A. 2013. The periodic capacitated arc routing problem with irregular services. Discrete Applied Mathematics. 161, (2013), 691-701. 